\title{
Full Electric Control of Exchange Bias
}

\author{
S. M. Wu, ${ }^{1,2, *}$ Shane A. Cybart, ${ }^{1,2}$ D. Yi, ${ }^{2,3}$ James M. Parker, ${ }^{1,2}$ R. Ramesh, ${ }^{1,2,3}$ and R. C. Dynes ${ }^{1,2,4}$ \\ ${ }^{1}$ Department of Physics, University of California, Berkeley, California 94720, USA \\ ${ }^{2}$ Materials Sciences Division, Lawrence Berkeley National Laboratory, Berkeley, California 94720, USA \\ ${ }^{3}$ Department of Materials Science and Engineering, University of California, Berkeley, California 94720, USA \\ ${ }^{4}$ Department of Physics, University of California San Diego, La Jolla, California 92093, USA
}

(Received 31 August 2012; published 4 February 2013)

\begin{abstract}
We report the creation of a multiferroic field effect device with a $\mathrm{BiFeO}_{3}$ (BFO) (antiferromagneticferroelectric) gate dielectric and a $\mathrm{La}_{0.7} \mathrm{Sr}_{0.3} \mathrm{MnO}_{3}$ (LSMO) (ferromagnetic) conducting channel that exhibits direct, bipolar electrical control of exchange bias. We show that exchange bias is reversibly switched between two stable states with opposite exchange bias polarities upon ferroelectric poling of the BFO. No field cooling, temperature cycling, or additional applied magnetic or electric field beyond the initial BFO polarization is needed for this bipolar modulation effect. Based on these results and the current understanding of exchange bias, we propose a model to explain the control of exchange bias. In this model the coupled antiferromagnetic-ferroelectric order in BFO along with the modulation of interfacial exchange interactions due to ionic displacement of $\mathrm{Fe}^{3+}$ in BFO relative to $\mathrm{Mn}^{3+/ 4+}$ in LSMO cause bipolar modulation.
\end{abstract}

DOI: 10.1103/PhysRevLett.110.067202

PACS numbers: $85.70 . \mathrm{Kh}$, 75.30.Et, 75.47.Lx

Exchange bias is a property of a coupled antiferromagnetic (AFM)-ferromagnetic (FM) system that occurs due to magnetic interface effects [1]. It is widely used in many applications, such as read heads in magnetic storage or spin valves [2]. Full control of exchange bias with electric field allows for an additional degree of freedom in applications and provides a path to the full electrical control of magnetization.

Despite its wide use, exchange bias is not completely understood [2-4], due to the technological limitations involved in directly observing and manipulating spin structure at an atomically thin magnetic interface. Recently, many groups have made progress in understanding the interfacial spin structure in exchange bias systems and several models of exchange bias have been proposed $[5,6]$. Here we report the direct electric field control of exchange bias from an applications perspective and as a technique for understanding exchange bias in light of recent findings.

Attempts at electric field control of exchange bias have been made in the past using various materials and devices, each having its own limitations for operation, and thus falling short of full direct electrical control of exchange bias. In $\mathrm{YMnO}_{3}$-Permalloy bilayers, it was possible to field cool a device and subsequently irreversibly suppress exchange bias [7]. Later, with $\mathrm{Cr}_{2} \mathrm{O}_{3}$ coupled to Pd-Co multilayers it was possible to reversibly switch between two different exchange bias polarity states through the application of electric field and an additional applied magnetic field after magnetoelectric field cooling [8].

In our previous work we showed the ability to reversibly switch between two exchange bias states with the same polarity (unipolar modulation) without the need for additional magnetic or electric fields in a multiferroic field effect device. This device used $\mathrm{BiFeO}_{3}$ (BFO), a multiferroic material, as the dielectric and $\mathrm{La}_{0.7} \mathrm{Sr}_{0.3} \mathrm{MnO}_{3}$ (LSMO), a ferromagnet, as the conducting channel [9]. Because of the coupled nature of the AFM and ferroelectric (FE) order parameters in $\mathrm{BFO}$, by reversing FE polarization in BFO, a simultaneous change should occur in the AFM spins at the interface, thus in principle allowing even further control of exchange bias [10]. Here we improve on our device and demonstrate the ability to reversibly switch between two exchange bias states with opposite polarity (bipolar modulation). We use the same structure but modify the direction of LSMO magnetization with respect to the current in the channel. A reversible shift of the polarity of exchange bias through the zero applied magnetic field axis is achieved with no magnetic or electric field cooling, no temperature cycling, and no additional electric or magnetic bias fields. Thus, without any additional caveats, we have achieved the full direct electric field control of exchange bias. Furthermore, because the modulation is bipolar, it suggests that pinned spins at the interface are reversing and that we have discovered a mechanism for affecting this change in a robust reproducible manner.

Using pulsed laser deposition, a 3-nm (8 unit cell) thick single crystal film of LSMO was grown on strontium titanate $\left[\mathrm{SrTiO}_{3}(001)\right]$, followed by a $200-\mathrm{nm}$ thick epitaxial layer of BFO. With argon ion milling and photolithography, the films were patterned into field-effect devices [Figs. 1(a) and 1(b)]. A detailed account of the film growth, film characterization, and device fabrication has previously been reported [9].

Devices were patterned with the conduction channel in both the [100] [Fig. 1(a)] and the [110] directions [Fig. 1(b)]. 


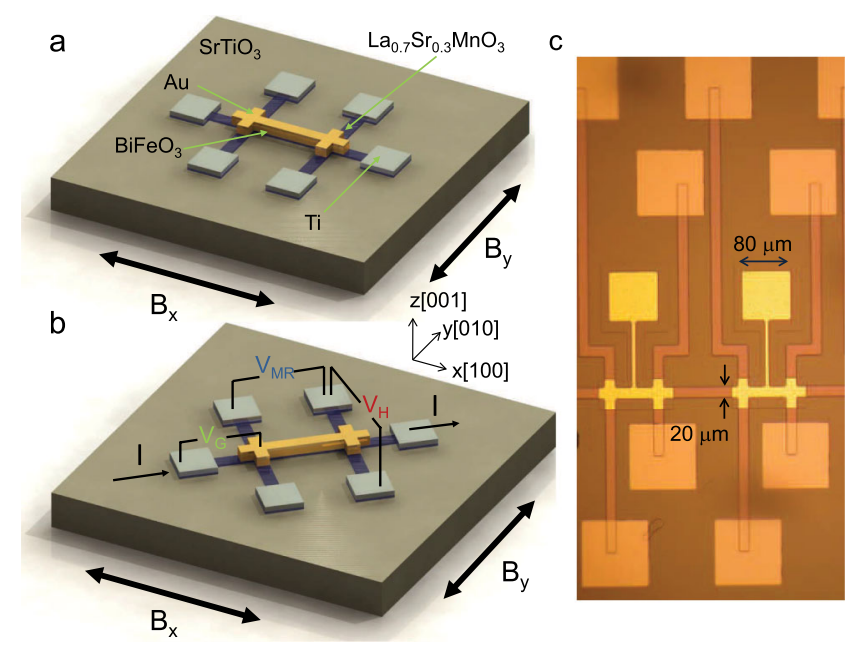

FIG. 1 (color online). (a), (b) Two devices with a gated hall bar geometry. In (a) current goes in the [100] direction, while in (b) the current goes along [110]. Voltage pulses $\left(V_{G}\right)$ are applied to the Au top gate electrode to ferroelectrically polarize BFO and the four point magnetoresistivity of LSMO is measured under applied fields in both the $x$ and $y$ directions. (c) An optical photograph of the device.

To investigate the magnetic properties of the LSMO channel, we measured its electrical resistance as a function of applied in-plane magnetic field in both the [100] $\left(B_{x}\right)$ and [010] $\left(B_{y}\right)$ directions, separately, as depicted in Figs. 1(a) and 1(b). Each magnetotransport measurement is performed at a static temperature, and at no point in the experiment was the device cooled in any electric or magnetic field. Typical measurements for both BFO FE polarizations are shown in Fig. S1 of the Supplemental Material [11]. The data exhibit magnetic hysteresis and have coercive and saturation features analogous to that of an $M-H$ hysteresis loop. We interpret the peaks in resistance as the coercive fields where there is zero net magnetization and that the tail is where magnetization becomes saturated. By determining these peak positions we determine the magnetic coercivity (distance between the peaks) and exchange bias (shift in the peaks off the origin) as shown in Fig. S1 [11]. Results using this technique are in excellent agreement with SQUID magnetometer $M-H$ measurements on unpatterned films and are further detailed in our previous work on such devices [9].

To examine the effect of BFO polarization on exchange bias, a $\pm 24 \mathrm{~V}$ voltage pulse was applied between the gate and the LSMO channel to polarize the BFO in the [001] direction. Measurements show that BFO polarization out of plane is fully saturated; however, complex domain structures may still form in plane since there are still four degenerate in-plane polarization states [9]. These structures are difficult to observe in our device due to the $\mathrm{Au}$ top electrode, but we can draw parallels from piezoresponse-force microscopy images for unpatterned BFO-LSMO heterostructures from previous work [9]. Magnetoresistance is measured and exchange bias is then determined using the above method. Multiple sweeps were performed and signal averaged for accuracy. This was then repeated for a sequence of pulses shown in Fig. 2(a). The nature of this experiment requires us to leave the LSMO magnetized in the positive or negative direction when returning to zero applied magnetic field before the next subsequent pulse. This remanent LSMO magnetization $\left(M_{R}\right)$ has a large effect on the exchange bias modulation, which we explore in this Letter.

Devices were patterned along both the [100] and the [110] directions with respect to the substrate [Figs. 1(a) and 1(b)] and magnetoresistance was measured with applied magnetic fields in both the [100] $\left(B_{x}\right)$ and the [010] $\left(B_{y}\right)$ directions separately. This provided four different device configurations for testing the angular dependence of exchange bias modulation. Devices with the measurement configuration of Fig. 1(a) with applied magnetic field $\left(B_{x}\right)$ exhibit unipolar exchange bias modulation consistent with previous work. In contrast, the three other device configurations exhibit bipolar modulation of exchange bias through zero magnetic field. The modulation behavior of these bipolar devices is remarkably similar, highly reproducible, and observed in 20 devices fabricated

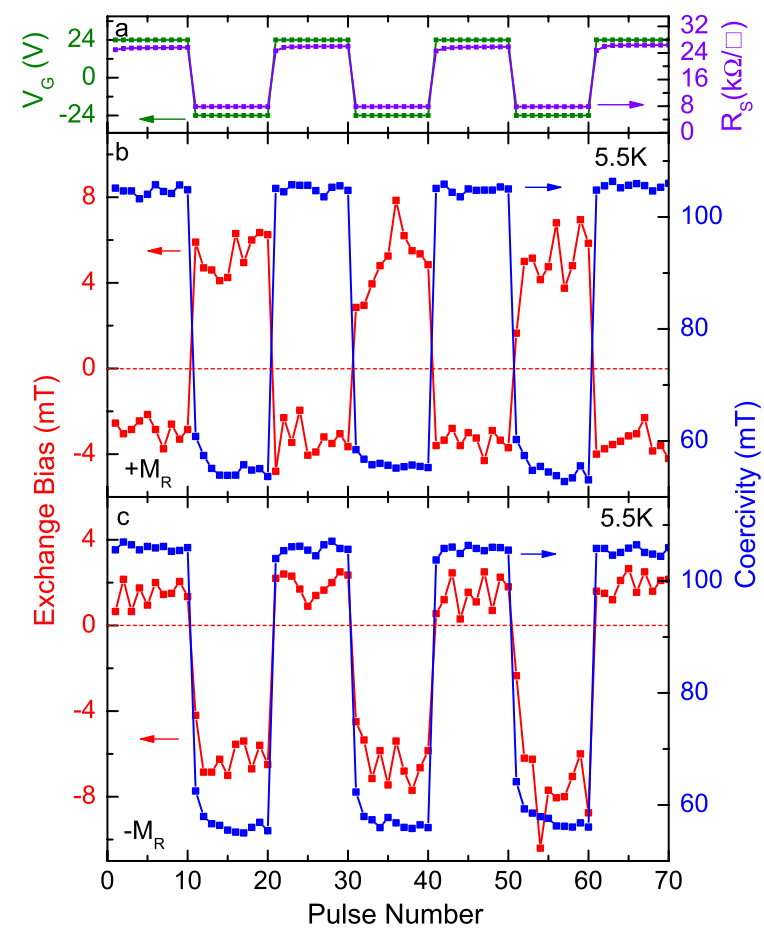

FIG. 2 (color online). (a) Gate pulse sequence applied before carrying out magnetoresistance measurements (green, left arrow) and the corresponding sheet resistance of the LSMO (purple, right arrow). (b), (c) Measurements of exchange bias and coercivity taken at $5.5 \mathrm{~K}$ after gate pulse shown in (a). Panels (b) and (c) represent the data when voltage pulses are applied in positive and negative remanent magnetization, respectively. Current was applied along the [110] direction with applied magnetic field in the $B_{x}[100]$ direction. 
on three separate epitaxially grown heterostructures. Figures 2(b) and 2(c) show bipolar modulation with respect to gate pulse at $5.5 \mathrm{~K}$ in both remanent magnetization states for a device patterned along the [110] [Fig. 1(b)] direction with magnetic field applied in [100] $\left(B_{x}\right)$. Depending on the remanent magnetization state of LSMO upon application of gate pulses, opposite FE polarizations lead to opposite directions of exchange bias. The modulation behavior is mirrored through the zero exchange bias axis between different remanent magnetization states. The temperature of the sample was held at $5.5 \mathrm{~K}$ throughout the measurement. The sole determining factors for the polarity of exchange bias in these bipolar devices are (a) the polarization state of the BFO film and (b) the $M_{R}$ of LSMO. To offer a comparison of the bipolar modulation behavior to unipolar modulation, a plot is presented in the supplemental figure Fig. S2 [11].

In addition to reversing exchange bias, the magnetic coercivity and channel resistance of LSMO also change with ferroelectric polarization. Supplemental Fig. S3 shows both effects with respect to temperature [11]. Pulsing the gate with a positive voltage we create a highresistance, high-coercivity state, while pulsing with a negative voltage creates a low-resistance, low-coercivity state. This is consistent at all temperatures measured and throughout all devices in all configurations regardless of the direction of applied magnetic field or device geometry relative to the crystal axes.

Temperature dependent measurements of exchange bias were taken. Following the same protocol outlined above, exchange bias in both remanent magnetizations was measured at different temperatures (Fig. 3). We observe that there is bipolar modulation in both remanent magnetization states of the LSMO with a decay towards zero exchange bias in both states as temperature is increased. The magnitude of exchange bias is always smaller in the positive BFO polarization state, which corresponds to the high electrical resistivity or magnetic coercivity state.

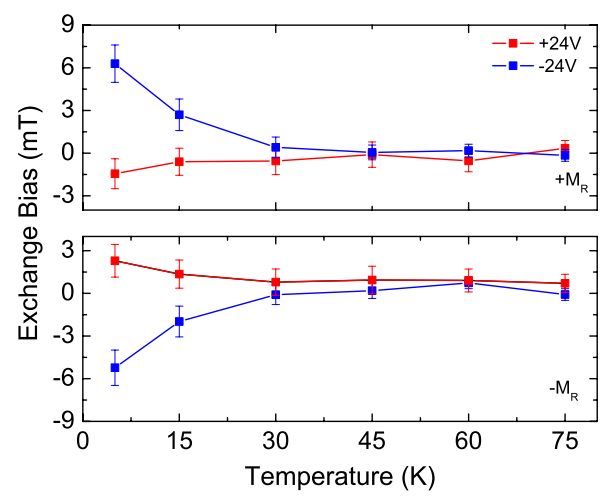

FIG. 3 (color online). Exchange bias as a function of temperature for both remanent magnetization states of LSMO for a device oriented in the [110] direction with magnetic field applied in the $B_{x}[100]$ direction.
Modulation disappears around $30 \mathrm{~K}$, well below the blocking temperature of this system which was previously determined to be around 100-120 K.

We can now speculate on the mechanism behind the electric field control of exchange bias in this system. The most recent exchange bias models are based on the existence of an interface state between the AFM and FM that is markedly different than either the FM or the AFM individually [12]. Such an interface state will produce two different types of spins that contribute unequally to the exchange bias system [13-16]. Some interface spins will become pinned in a single direction by the AFM, unaffected by magnetic field. The interaction between these pinned spins and the FM causes an effective "bias field" that the underlying FM layer must overcome before magnetization can be switched, resulting in exchange bias. Other interface spins will rotate with the FM layer when the magnetic field is swept, because they are coupled more tightly to the FM than the AFM. This results in a spin drag effect that increases the coercivity of the FM material. Several groups have observed these types of magnetic interface interactions [5,6]. Previous work showed an emergent interfacial magnetic state, correlated with exchange bias, in BFO-LSMO heterostructures resulting from the enhanced canting of the AFM spins at an otherwise magnetically compensated interface [17]. Furthermore, in this system the coupling between $\mathrm{Fe}^{3+}$ in the magnetic interface layer and the $\mathrm{Mn}^{3+/ 4+}$ was antiferromagnetic. Many exchange bias systems exhibit this type of AFM interfacial coupling, but no differences in exchange bias occur until extremely high magnetic fields are applied to overcome the interfacial coupling and align all spins to the external magnetic field. Since we do not use such high fields, the exchange bias model described above does not change. Some spins will be pinned, and some will rotate with the FM; since the coupling to the interface layer is antiferromagnetic, the interface spins will evolve in the opposite fashion as compared to the ferromagnetically coupled case [18].

Based on these models we present an explanation for the following effects: bipolar exchange bias modulation, the remanent magnetization dependence of that modulation, and coercivity modulation. The progression of the magnetic state in our device as magnetic field is swept and after a gate pulse reverses BFO polarization is shown in Fig. 4. Figure 4(a) shows the progression in terms of a magnetic hysteresis loop and Fig. 4(b) shows it from an interfacial spin state perspective. Because of the ferroelectric nature of BFO, when ferroelectric polarization is switched, the Fe and $\mathrm{Bi}$ ions will move relative to the oxygen octahedra [19]. Since the LSMO channel does not exhibit the same effect, the $\mathrm{Fe}$ ion at the interface in BFO exists in two different states depending on FE polarization, one closer to the LSMO channel and one farther away. The AFM anisotropy is much greater than the FM anisotropy [20,21]; 

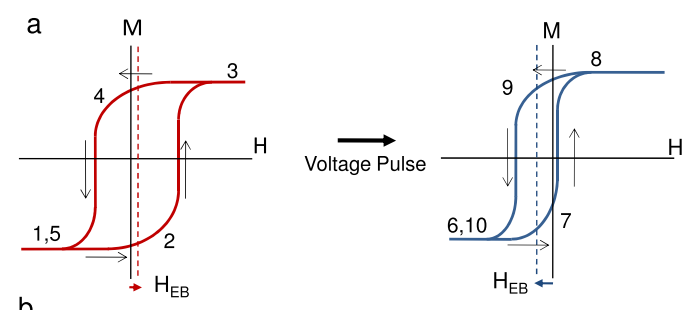

b

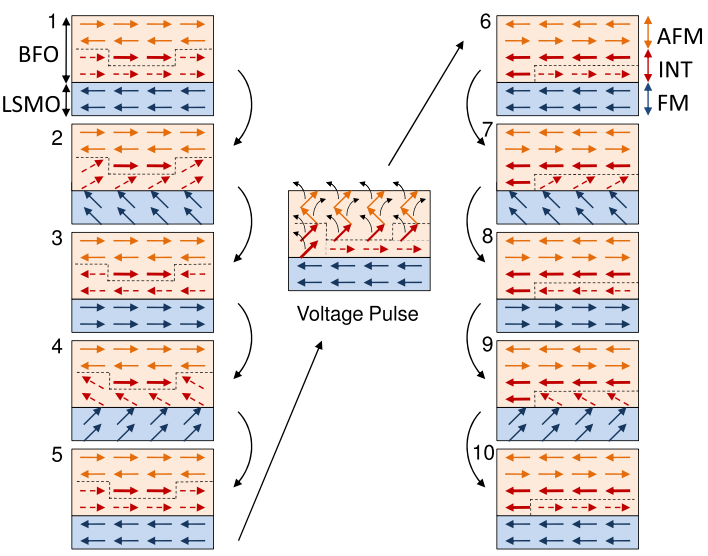

FIG. 4 (color online). (a) Magnetic hysteresis curve of LSMO before and after BFO ferroelectric polarization reversal. Numbers and arrows represent the progression of time as magnetic field is swept. (b) Depiction of interfacial spins for each number in (a). AFM anisotropy is reduced at the interface between a FM (orange, top) and AFM (blue, bottom) creating an interface layer (red, center) with rotatable AFM spins (dashed red arrows) that rotate with the FM when magnetic field is applied and pinned AFM spins (bold red arrows) that are not affected by applied magnetic field.

thus, we are left with only two parameters that determine the exchange bias behavior: AFM anisotropy $\left(K_{\mathrm{AFM}}\right)$ and the interfacial interaction energy $\left(J_{\mathrm{AFM}-\mathrm{FM}}\right)$. By changing the distance between interacting ions, we are manipulating $J_{\mathrm{AFM}-\mathrm{FM}}$ relative to $K_{\mathrm{AFM}}$ causing two different exchange bias states to exist: the high $J_{\mathrm{AFM}-\mathrm{FM}}$ (positive gate voltage pulse, down polarization, high resistivity, $\mathrm{Fe}$ closer to interface) state and the low $J_{\mathrm{AFM}-\mathrm{FM}}$ (negative gate voltage pulse, up polarization, low resistivity, $\mathrm{Fe}$ farther from interface) state. Initially [Fig. 4(b)(1)] the system is in the negative magnetization state, with small magnitude positive exchange bias and high coercivity. As magnetic field is swept [Fig. 4(b)(1-5)] a large number of interfacial spins rotate with the FM while a smaller fraction remain pinned, this results in the high-coercivity-lowexchange bias state and is caused by increased coupling to the FM due to ionic displacement. After applying a negative voltage pulse to the gate and changing ferroelectric polarization, we are now in a different regime and the device now exhibits high magnitude negative exchange bias and low coercivity [Fig. 4(b)(6)]. Since the coupling at the interface is weakened due to ionic displacement, a larger fraction of interfacial spins are pinned to the AFM. Because of the coupled AFM-FE order in BFO, the individual coupled spins have also changed polarity leading to exchange bias reversal.

In all our devices, changing between remanent magnetization states required positive gate pulses before negative pulses. By reversing the direction of sweeping, exchange bias behavior does not significantly change even upon further negative pulsing. Using the same model, we explain this effect and the remanent magnetization dependence. A positive voltage pulse is first required to increase coupling to the FM so more interfacial spins rotate with the LSMO upon sweeping. After sweeping the magnetic field, the rotatable spins will now be in the direction of the LSMO remanent magnetization, and once a negative voltage pulse is applied, some fraction of these rotatable spins become pinned, contributing to exchange bias in the exact opposite direction.

The similarity of modulation behavior between the three bipolar configurations suggests that the unipolar device is a special case, and there might exist a mechanism that nullifies the bipolar behavior. In fact, as we show in supplemental Fig. S2 [11], the high-coercivity state in the unipolar device has the same exchange bias polarity and magnitude as the bipolar device. This suggests that the model for exchange bias modulation in the unipolar devices is still the same, but the effect of pinned spins reversing under BFO polarization switching is absent. The exchange bias magnitude is still larger in the low-coercivity state, which suggests that there is still an increase in pinned spins but the direction is solely determined by the magnetization of the LSMO and is not flipped by BFO polarization. Reasons for this lack of spin reversal are not well understood although they may be related to the BFO ferroelectric domain structure in plane, which can only be imaged on the unpatterned heterostructures at room temperature before device fabrication and thus remains a mystery to us after numerous switches. Changes in the domain structure could favor a certain type of switching behavior that leaves AFM interface spins untouched upon switching.

We have discovered a mechanism for the direct control of exchange bias with electric field. This effect is reversible and comes concurrently with the modulation of channel resistance (sometimes over 300\%) and the modulation of magnetic coercivity. This type of exchange bias control is a crucial first step for fully controlling the magnetization of a thin film using an electric field [22,23]. Using this method of controlling magnetization would offer a low-current or low-power alternative to the typical current induced magnetization control mechanisms [24]. From both a device applications and physics standpoint, these results represent an exciting advance for the next generation of exchange bias systems and devices.

This work was supported by the U.S. Department of Energy under Contract No. DEAC02-05CH11231, NSFE3S Engineering Research Center, and the DARPA Spin Logic Program. 
*stephenw@berkeley.edu

[1] W. H. Meiklejohn and C.P. Bean, Phys. Rev. 102, 1413 (1956).

[2] J. Nogues and I. K. Schuller, J. Magn. Magn. Mater. 192, 203 (1999).

[3] M. Kiwi, J. Magn. Magn. Mater. 234, 584 (2001).

[4] A. E. Berkowitz and K. Takano, J. Magn. Magn. Mater. 200, 552 (1999).

[5] H. Ohldag, A. Scholl, F. Nolting, E. Arenholz, S. Maat, A. T. Young, M. Carey, and J. Stohr, Phys. Rev. Lett. 91, 017203 (2003).

[6] J. Wu, J. S. Park, W. Kim, E. Arenholz, M. Liberati, A. Scholl, Y.Z. Wu, C. Hwang, and Z. Q. Qiu, Phys. Rev. Lett. 104, 217204 (2010).

[7] V. Laukhin, V. Skumryev, X. Marti, D. Hrabovsky, F. Sanchez, M. V. Garcia-Cuenca, C. Ferrater, M. Varela, U. Luders, J. F. Bobo, and J. Fontcuberta, Phys. Rev. Lett. 97, 227201 (2006).

[8] X. He, Y. Wang, N. Wu, A. N. Caruso, E. Vescovo, K. D. Belashchenko, P. A. Dowben, and C. Binek, Nat. Mater. 9, 579 (2010).

[9] S. M. Wu, S. A. Cybart, P. Yu, M. D. Rossell, J. X. Zhang, R. Ramesh, and R. C. Dynes, Nat. Mater. 9, 756 (2010).

[10] T. Zhao, A. Scholl, F. Zavaliche, K. Lee, M. Barry, A. Doran, M. P. Cruz, Y.H. Chu, C. Ederer, N. A. Spaldin, R. R. Das, D. M. Kim, S.H. Baek, C. B. Eom, and R. Ramesh, Nat. Mater. 5, 823 (2006).

[11] See Supplemental Material at http://link.aps.org/ supplemental/10.1103/PhysRevLett.110.067202 for supplementary figures.
[12] Magnetic Heterostructures, Springer Tracts in Modern Physics Vol. 227, edited by F. Radu and H. Zabel (Springer, Berlin, 2008), p. 97.

[13] J. Nogues, J. Sort, V. Langlais, V. Skumryev, S. Surinach, J. Munoz, and M. Baro, Phys. Rep. 422, 65 (2005).

[14] B. Miller and E. Dahlberg, Appl. Phys. Lett. 69, 3932 (1996).

[15] M. D. Stiles and R. D. McMichael, Phys. Rev. B 59, 3722 (1999).

[16] M.D. Stiles and R. D. McMichael, Phys. Rev. B 63, 064405 (2001).

[17] P. Yu, J.-S. Lee, S. Okamoto, M. D. Rossell, M. Huijben, C.-H. Yang, Q. He, J. X. Zhang, S. Y. Yang, M. J. Lee, Q. M. Ramasse, R. Erni, Y.-H. Chu, D. A. Arena, C.-C. Kao, L. W. Martin, and R. Ramesh, Phys. Rev. Lett. 105, 027201 (2010).

[18] J. Nogues, C. Leighton, and I. K. Schuller, Phys. Rev. B 61, 1315 (2000).

[19] J. X. Zhang, Q. He, M. Trassin, W. Luo, D. Yi, M. D. Rossell, P. Yu, L. You, C. H. Wang, C. Y. Kuo, J. T. Heron, Z. Hu, R. J. Zeches, H. J. Lin, A. Tanaka, C. T. Chen, L. H. Tjeng, Y.H. Chu, and R. Ramesh, Phys. Rev. Lett. 107, 147602 (2011).

[20] C. Ederer and N. A. Spaldin, Phys. Rev. B 71, 060401 (2005).

[21] K. Steenbeck and R. Hiergeist, Appl. Phys. Lett. 75, 1778 (1999).

[22] R. Ramesh and N. A. Spaldin, Nat. Mater. 6, 21 (2007).

[23] J. Ma, J. M. Hu, Z. Li, and C. W. Nan, Adv. Mater. 23, 1062 (2011).

[24] S. Zhang, P. M. Levy, and A. Fert, Phys. Rev. Lett. 88, 236601 (2002). 